\title{
Statistical Study of North-South Asymmetry during Solar Cycles 21, 22, 23 and 24
}

\author{
Raj Kumar, Mahesh Chandra Mathpal, Bimal Pande and Seema Pande* \\ Department of Physics (UGC-Centre of Advanced Study), Kumaun University, Nainital, India. \\ Email: pande.seema@yahoo.com
}

\begin{abstract}
North-south asymmetry has been a significant aspect exhibited by various solar activity parameters of the Sun. In this work we have statistically analyzed soft X-ray (SXR) flares during the period Jan 1981 to Dec 2016 for four different solar cycles (SCs) covering cycles 21, 22, 23 and 24. We have presented the class wise (B, C, M \& X) variation of SXR flares for these SCs. SXR flare activity is lowest in solar cycle 24 as compared to cycles 22 and 23. It is shown that $\mathrm{M}$ class activity continuously decreased from SC 21 to 24 . X and C class flares show higher activity in SC 22 and 23 as compared to SC 24, whereas B class flare activity is higher for SC 23. We have also studied the north-south (N-S) asymmetry of SXR flares for SCs 21, 22, 23 and 24. Our study revealed that during SCs 21, 22 and 23 the flare activity was more pronounced in the Southern hemisphere. However, the flare activity was equally distributed between the northern and southern hemispheres for SC 24.
\end{abstract}

Keywords: Solar Cycles, North-South Asymmetry, Solar activity.

\section{Introduction}

Magnetic activity in the solar atmosphere changes over every solar cycle, therefore most of the solar activity features such as sunspot number and solar X-ray flares show periodic variations. The solar activity phenomena demonstrate non-uniform distribution between northern and southern hemispheres of the solar disc. This phenomenon is called N-S asymmetry. There are many papers wherein various authors have studied the N-S asymmetry of solar activity features like: solar $\mathrm{X}$-ray flares, sunspot numbers, sunspot area and solar active prominences etc. N-S asymmetry of soft X-ray flares was studied by Garcia (1990) for the SC 20 and 21 who concluded that northern hemisphere is more dominant during the early part of the cycle and dominancy goes to the southern hemisphere as the cycle progresses. Li et al. (1998) have shown that N-S asymmetry of SXR flares favored the southern hemisphere during SC 22. Pande et al. (2009) studied N-S asymmetry of SAP data during SC 23 and inferred that activity in both of the hemispheres equally dominates during the rising phase of the cycle and after 1999 activity in the southern hemisphere dominates. Pande et al. (2010) studied the SXR flares statistically for the SC 21, 22 and 23 and found that during SC 21, 22, 23 activity was southern hemisphere dominated. They also found that during the minimum activity of SCs, B class flares were more frequent whereas other flare classes dominated for the maximum activity of SCs.

In this paper we have analysed the total number of SXR flares from SCs 21 to 24. Total SXR flare distribution is compared with different classes of SXRs (class B, C, M and $\mathrm{X}$ ). We have also analysed the N-S asymmetry of SXR flares for solar cycles 21 to 24. In section 2, data set and statistical analysis methods are described. Section 3 contains the description of SXR distribution and N-S asymmetry. In section 4 , results are described. 


\section{Data set and statistical method}

For the present analysis, we have taken the data from national geophysical data centre (NGDC) anonymous ftp server: ftp://ftp.ngdc.noaa.gov/STP/space-weather/ solar-data/solar-features/solar-flares/X-rays/ goes/xrs.

We have analysed the data from January 01, 1981 to December 31, 2016. For the analysis of $\mathrm{N}-\mathrm{S}$ asymmetry, we have considered only that data for which latitude angles in northern and southern hemisphere are given.

To calculate the N-S asymmetry index $\left(\mathrm{A}_{\mathrm{NS}}\right)$, we have used the following formula (Pande et al., 2010):

$A_{N S}=\frac{N-S}{N+S}$

Where $\mathrm{N}$ and $\mathrm{S}$ are the number of SXR flare events observed in northern and southern hemispheres of the solar disk respectively. If $A_{N S}$ is positive, then activity in northern hemisphere dominates and if it comes out to be negative, activity in southern hemisphere is more pronounced.

\section{Analysis}

Fig.1 shows the yearly variation of SXR flares for different solar cycles. The first curve shows a periodical variation of total SXR flares with each SC. Class C flares and total SXRs vary in the same manner while class B flares exhibit a different behavior. In Fig. 2, N-S asymmetry of SXR flares is shown for different SCs.

\section{Results and Discussion}

Based on the statistical analysis of SXR flare data from January, 1981 to December, 2016 the following results are obtained:

- The SXR flares, plotted for different SCs (first plot of Fig. 1) show a periodic variation with the solar cycles. Maximum SXR flare counts continuously decrease from cycle 21 to 24 . These are lowest for the SC 24. For the decaying phase of the cycle 21 and rising phase of the cycle 22 , class B flares show an opposite behavior to the total SXR flares, which suggests that class B flare do not provide a significant contribution to the total SXR flares during this range of the cycles.

- The plot of Class C flares versus years matches with the plot of total SXR flares versus years which implies that class $\mathrm{C}$ flares provide considerable contribution to the total SXR flares. Class M stipulates considerable amount of contribution to the total SXR flares for the decaying phase of SC 21. But after that it shows much less contribution for the further cycles and decreases gradually with the increasing SCs. Class $\mathrm{X}$ flares show periodic random variations with the SCs. Their count is highest for the SC 22 and these are less in number as compared to $\mathrm{B}, \mathrm{C}$ and $\mathrm{M}$ class flares but since these are most energetic SXR flares so X class flares play important role in the energy consideration of SXR flares.

- Fig.2 shows the N-S asymmetry of total SXR flares for different SCs. From SC 21 to 23 activity in the southern hemisphere is dominant. It means maximum of the SXR flares are obtained from southern hemisphere of the solar disc. For cycle 24, activity in both the northern and southern hemispheres is approximately equally distributed. 

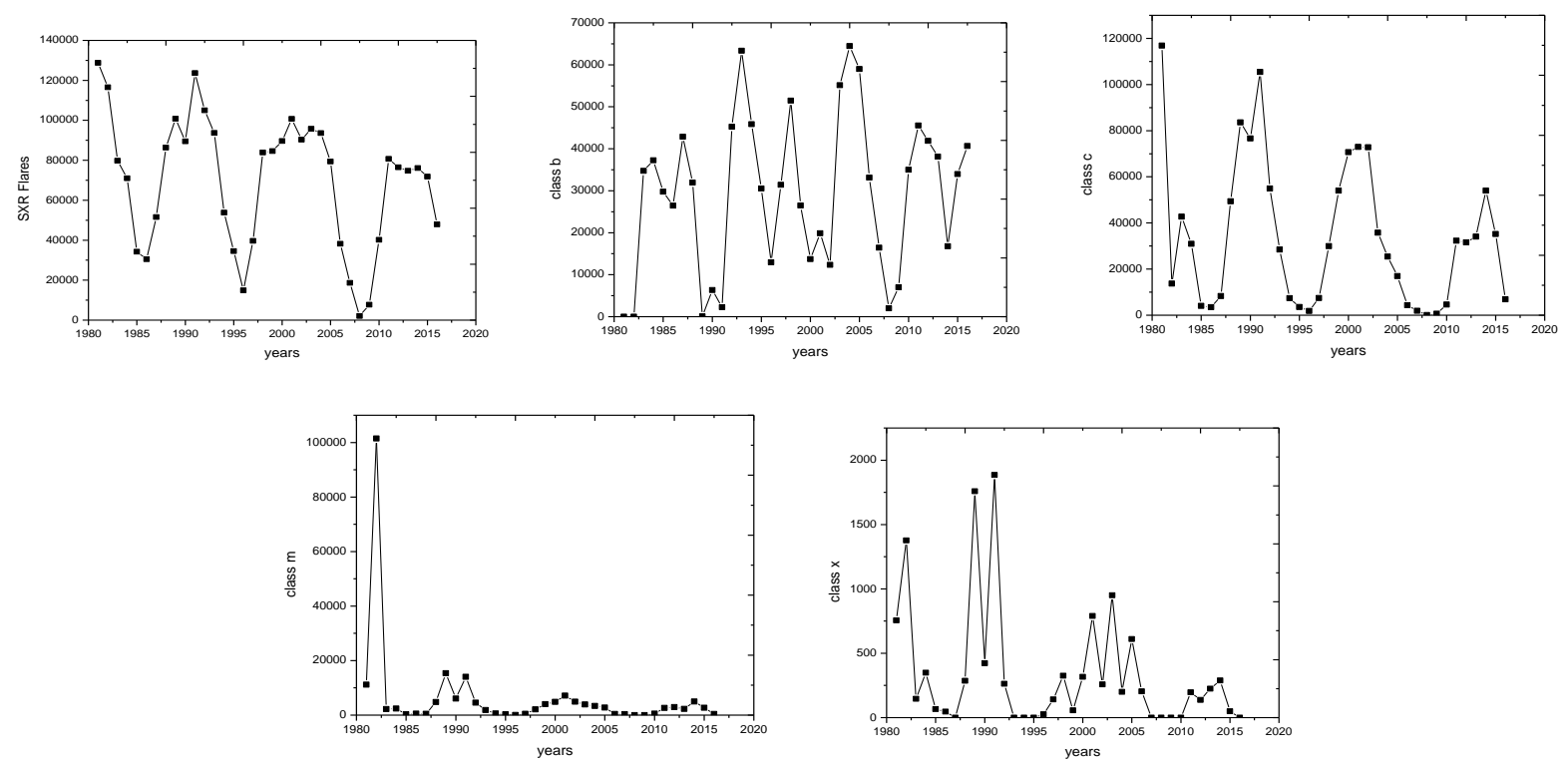

Fig.1: Yearly plot of total SXR flares and different classes of flares (class B, C, M and X).

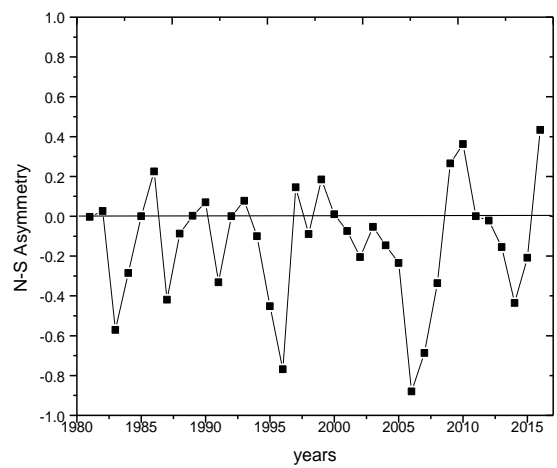

Fig.2. Plot of N-S asymmetry indices for total SXR flares versus years

\section{References:}

Garcia, H.A., 1990. SoPh, 127, 185.

Joshi, B., Joshi, A., 2004. SoPh 219, 343.

Joshi, N.C., Bankoti, N.S., Pande, S., Pande, B., Pandey, K., 2009. SoPh 260, 451.

doi:10.1007/s11207-009-9446-2.

Joshi, N.C., Bankoti, N.S., Pande, S., Pande, B., Uddin, W., Pandey, K., 2010.Elsvier

B.V.1384-1076.
Li, K.-J., Schmieder, B., Li, Q.-Sh., 1998. A\&AS 131, 99. 